\title{
Oscillatory activity in prefrontal and posterior regions during implicit letter-location binding
}

\author{
Pablo Campo ${ }^{a, *+1}$, Claudia Poch ${ }^{a, 1}$, Fabrice B.R. Parmentier ${ }^{b, c, d}$, Stephan Moratti ${ }^{\text {, }}$, Jane V. Elsley ${ }^{d}$, \\ Nazareth P. Castellanos ${ }^{e}$, José María Ruiz-Vargas ${ }^{f}$, Francisco del Pozo ${ }^{\text {e }}$, Fernando Maestú a,g \\ a Center of Magnetoencephalography Dr. Rérez-Modrego, Compintense University of Madrid, Madrid, Spain \\ b Clinical Neuropsychology Group. Department of Psychology and Heaith Sciences Research Institute, University of the Balearic Islands, Spoin \\ "School of Psycholosy, University of Western Australia \\ a school of Pyychology, University of Pyymouth \\ e Center of Bionedical Technology (CTB). Polytechnic University of Madrid, Madrid, Spain \\ $r$ Department of Basic Psychology, University Autonona of Madrid, Madrid, Spain \\ s Departnent of Basic Psychology Cognitive Processes ii, Compiutense University of Madrid, Madrid, Spain
}

\begin{abstract}
A B S T R A C T
Many cognitive abilities involve the integration of information from different modalities, a process referred to as "binding." It remains less clear, however, whether the creation of bound representations occurs in an involuntary manner, and whether the links between the constituent features of an object are symmetrical. We used magnetoencephalography to investigate whether oscillatory brain activity related to binding processes would be observed in conditions in which participants maintain one feature only (involuntary binding): and whether this activity varies as a function of the feature attended to by participants (binding asymmetry). Participants performed two probe recognition tasks that were identical in terms of their perceptual characteristics and only differed with respect to the instructions given (to memorize either consonants or locations). MEC data were reconstructed using a current source distribution estimation in the classical frequency bands. We observed implicit verbal-spatial binding only when participants successfully maintained the identity of consonants, which was associated with a selective increase in oscillatory activity over prefrontal regions in all frequency bands during the first half of the retention period and accompanied by increased activity in posterior brain regions. The increase in oscillatory activity in prefrontal areas was only observed during the verbal task, which suggests that this activity might be signaling neural processes specifically involved in cross-code binding. Current results are in agreement with proposals suggesting that the prefrontal cortex function as a "pointer" which indexes the features that belong together within an object.
\end{abstract}

Working Memory (WM) is considered a supporting process for high level cognitive abilities (Miyake and Shah, 1999), many of which require the combination and maintenance of complex information, demanding cross-code associations (Cowan et al., 2006). Despite its crucial role in a number of mencal skills and abilities, visual WM demonstrates, in humans, a surprisingly limited capacity, estimated at around three to four units of information, while at the same time suggesting that the term "unit" pertains not to individual features, but objects (Cowan, 2001; Vogel and Machizawa, 2004; Vogel et al., 2001; Zhang and Luck, 2008). Additionally, other authors have suggested the additional role of visual complexity to limited capacity (Alvarez

\footnotetext{
* Corresponding author. Centro de Magnetoencefalografia Dr. Pérez-Modrego. Pabellón 8, Facultad de Medicina, Universidad Complutense de Madrid. 28040, Madrid, Spain. Fax: +34913942294.

E-mail address: pcampo@iparakis.es (P. Campo).

1 These authors contributed equally to the study.
}

and Cavanagh, 2004, although see Eng et al., 2005). More recently, Bays and Husain (2008) have proposed that limited capacity is better explained by a limited resource distributed across all items in the scene, and not by the number of items per se. Consequently, the integration of features into coherent objects has been increasingly recognized as a critical determinant of memory performance (Cowan, 2001). A good part of the existing research on binding and WM has focused on the integration of visual features (Bodelon et al., 2007; Friedman-Hill et al., 1995; Luck and Vogel, 1997; Todd and Marois, 2005; Zhang and Luck, 2008) and, to a smaller extent, auditory features (Maybery et al., 2009; Saito et al., 2005; Widmann et al., 2007). Despite decades of research focusing on the functional distinction between verbal and visuo-spatial represencations (Baddeley, 1996; Baddeley and Hitch, 1974; Logie, 1995; Miyake and Shah, 1999; Smith and Jonides, 1998), surprisingly little attention has been devoted to the integration of verbal and spatial information. However, in real-world scenes we are faced daily with this kind of integrated 
information in the form of traffic signals, or tracking routes on maps, etc. Nonetheless, some progress has been made in underscanding the behavioural mechanisms (Cowan et al., 2006; Elsley and Parmentier, 2009; Kahneman et al., 1992; Mitroff and Alvarez, 2007; Morey, 2009; Oberauer and Vockenberg, 2009) and the neural bases (Campo et al., 2008; Campo et al., 2005; Luck et al., 2008; Prabhakaran et al., 2000; Wu et al., 2007) of verbal-spatial binding.

While evidence supports the notion of bound representations, the exact mechanisms underpinning binding in WM remain relatively unspecified, in part because some key questions remain unanswered. Two issues have recently arisen that may constrain the development of a mechanistic model of binding: the role of attention and the asymmetrical nature of binding. Several lines of research suggest that selective attention is involved in binding the constituent features of objects (Duncan, 1984; Martinez-Trujillo and Treue, 2004; Scholl, 2001; Treisman, 1998; Vecera and Farah, 1994). However, whether binding occurs in an involuntary manner remains unclear. Several studies have shown that in the absence of attention visual features can be represented jointly (Humphrey and Goodale, 1998; Kahneman et al., 1992; Mazza et al., 2007; Meegan and Honsberger, 2005; Melcher et al., 2005; O'Craven et al., 1999; Schoenfeld et al., 2003; Sohn et al., 2004; see also Constantinidis and Steinmetz, 2005). Consequently, it has been proposed that attentional processes directed to task-relevant features of an object automatically spreads to other irrelevant features of the attended object leading to a bound represencations of "spatiotemporally colocalized features" (Melcher et al., 2005; Sohn et al., 2004). Thus, according to this position, attendance to a task-relevant object feature may entail the involuntary binding of all features of that object.

A second and related issue of importance is that regarding the nature of binding, and more particularly whether binding is best described by full integration of features or by asymmetric links between features (Maybery et al., 2009). There is indeed evidence suggesting that not all features are of equal importance in the formation of integrated representations (Hommel and Colzato, 2004). For example, spatial information appears to play a primordial role in visual WM, resulting in the automatic processing of location following the encoding of a visual feature, such as shape or color (jiang et al., 2000; Mazza et al., 2007; Meegan and Honsberger, 2005; Olson and Marshuetz, 2005; see also Conscantinidis and Steinmetz, 2005). An analogous, yet opposite asymmetry is observed in the auditory domain, where pitch is proposed as a primary dimension compared to location information (Kubovy and Van Valkenburg, 2001; Maybery et al., 2009). The asymmetrical nature of binding was further investigated by Elsley. Parmentier and Maybery (unpublished results). Their Experiment 2 made use of an adapted version of the single probe task developed by Prabhakaran et al. (2000). Participants were presented with an array of three irregular black polygon shapes (simultaneously) in distinct locations in the screen. In one task, participants were required to remember the visual (shape) features only: in the other they were required to remember the spatial (locations) features only. At test, they had to decide whether a single probe shape in location represented a shape they had seen in the memory display (the visual task) or a location that was occupied in the memory display (the spatial cask). In both tasks, performance was compared across two critical probe conditions, both of which required a "yes" response. Intact probes consisted of a shape in the location it was originally, in the memory display. Recombined probes consisted of a shape in a location originally occupied by a different to-beremembered shape. The logic was simple: if participants cannot retrieve one feature independently of the other (i.e., the features were bound in memory), there should be a performance advantaged in the intact condition where the original bindings were preserved relative to the recombined condition, where they were not-i.e., a "binding effect" should be observed. Their results indicated a significant binding effect in the visual task (where shapes only were attended) but not in the spatial task (where the locations only were attended to). Additionally, in their Experiment 3, they examined whether the binding of locations to shapes was mediated by the level of discriminability of the shape features. The hypothesis was that the relative distinctiveness of the shapes may have determined the extent to which spatial locations would be bootstrapped to them in order to form more distinguishable objects. Comparisons across easily distinguishable and less distinguishable shape sets in a "visual task" (as above) indicated, however, that the binding of locations to shapes was not impacted by the degree to which the shapes themselves could be distinguished. Taken together, these findings support asymmetry such that attending to an aspect of an objects' identity encails binding to its spatial location, while attending to spatial location does not necessarily entail its binding to the item's identity (see also Constantinidis and Steinmetz, 2005; Jiang et al., 2000).

When considering verbal-spatial integration, neither the symmetry of binding nor the automaticity of binding have been examined from a neural perspective. We were interested in brain activity during maintenance period as it is considered as a process of keeping information in mind in the absence of external stimuli, and corresponds to the mnemonic aspect of the WM. Investigating this phase could provide information about the neural mechanisms underlying bound information represencation. Accordingly, in the present study, we used magnetoencephalography (MEG) to measure brain activity underpinning the maintenance of verbal and spatial features in two recognition casks, based on a letter-location paradigm previously used in binding studies (Prabhakaran et al., 2000). In the verbal task participants attended consonants, while in the spatial task they attended locations. The aim of the current study was to elucidate whether neural activity specific to binding would be observed in conditions in which participants attend to one feature only (involuntary binding); and whether the brain activiry underpinning binding processes varies as a function of the feature attended to by participants (binding asymmetry). In both the verbal and spatial tasks, participants were presented with four consonants appearing simultaneously in four distinct locations. Thus both tasks were identical in terms of their perceptual characteristics and only differed with respect to the instructions given to participants (to memorize either the consonants or the locations). Of critical importance to measure binding (Oberauer and Vockenberg. 2009), positive-recognition probes could either be made up of a letter that was in location (incact probe) or a letter and a location that were both in the array, but were not seen together (re-combined probe). If verbal and spatial features are integrated in WM, accuracy and/or reaction time (RT) should show an advantage for incact probes as compared to recombined probes (in line with past research; Prabhakaran et al., 2000; Elsley and Parmentier, 2009). Henceforth, we refer to this as the "binding effect." In contrast, if the task-irrelevant feature, either verbal or spatial, is not concurrently maintained, intact and recombined probes should be functionally equivalent and yield similar levels of performance. Based on previous observations that encoding the "what" appears to encail the involuntary encoding of the "where" (Bao et al., 2007; Mazza et al., 2007; Meegan and Honsberger, 2005), we hypothesized that location information should be automatically processed when participants attend the consonants, resulting in a binding effect. No such binding effect was expected when location was the relevant feature, however. Similar to the results obtained in previous neuroimaging studies of visual-feature binding (Donner et al., 2002; Shafritz et al., 2002; Todd and Marois, 2004; Vogel and Machizawa, 2004; $X u$ and Chun, 2006), findings from functional magnetic resonance imaging (IMRI) and MEG/EEG studies highlight prefrontal and parietal regions as the neuroanatomical correlates of verbal-spatial binding (Campo et al., 2008; Campo et al., 2005; Luck et al., 2008; Prabhakaran et al., 2000; Wu et al., 2007). In essence, these previous studies provide haemodynamic or evoked electrical/ magnetic correlates of binding without addressing the physiological mechanisms underlying this process. An alternative approach 
proposes that neural oscillations might be a potential mechanism by which separate features of an object are bound (Singer, 1999; Varela et al., 2001). Specifically, this approach considers that neurons coding various features of objects are bound into a neuronal assembly by synchronization of their activity and has been supported by numerous studies (Doesburg et al., 2008b; Engel et al., 2001; Palva et al., 2005; Senkowski et al., 2006; Womelsdorf et al., 2007). Although neural oscillatory activity in the gamma band was originally proposed as the mechanism underlying binding processes (Singer, 2001; Singer and Gray, 1995; Tallon-Baudry and Bertrand, 1999), oscillations in other "classical" frequency ranges (theta, alpha and beta) have also been linked with visual-feature binding and cross-modal binding (Bassett et al., 2006; Hummel and Gerloft, 2005; Mima et al., 2001; Palva et al., 2005; Wu et al., 2007). While it has been shown that WM operations engage oscillations in different frequency ranges (Grimault et al., 2009; Jokisch and Jensen, 2007; Sauseng et al., 2004; Tesche and Karhu, 2000), the actual role of each frequency in representing the contents of WM remains unclear (Kahana, 2006; Palva et al., 2005). As the neural dynamics required for cross-code integrated representations presumably demands interactions between activities of multiple brain regions in different frequency ranges (Calvert et al., 2000; Doesburg et al., 2008a; Palva et al., 2005), here, we propose that neuronal oscillatory activity in various frequencies should be greater when verbal and spatial features are maintained as an integrated represencation.

Accumulated evidence from psychophysical, functional neuroimaging and transcranial magnetic stimulation studies strongly support the crucial role of posterior parietal cortex in visual-feature integration processes (Corbetta et al., 1995; Donner et al., 2002; Lee and Chun, 2001; Shafritz et al., 2002; Simon-Thomas et al., 2003; Todd and Marois, 2004, 2005; Vogel and Machizawa, 2004; Xu and Chun, 2006) and verbal-spatial binding (Campo et al., 2008; Campo et al., 2005; Wu et al., 2007). The prefrontal cortex has been proposed to integrate object identity and location through connections within prefrontal areas (Prabhakaran et al., 2000; Rao et al., 1997) and through converging projections from parietal and temporal regions on the prefrontal cortex (Fuster, 1995; Rao et al., 1997). These previous results have led us to hypothesize that greater oscillatory activity might be observed in prefrontal and pariecal regions when concurrent verbal-spatial maintenance occurs.

Thus, the predictions of the present study were as follows: first, from a behavioural perspective, if verbal and spatial binding is characterised by binding asymmetry (as is reported elsewhere for visual and spatial features e.g., Elsley et al., unpublished results; Jiang et al., 2000) we should observe a binding effect (intact over recombined probe advancage) when item (consonant) identicy is task relevant, but not when spatial location is task-relevant. Crucially however, the former but not the latter should be characterised by greater oscillatory activicy within prefrontal and parietal regions.

\section{Materials and methods}

Subjects

Fourteen adult subjects (mean age 25.36 years, SD of 3.13 years, range 22 years to 32 years, nine female), without any history of neurological or psychiatric illness, volunteered for participation in the study and gave written consent, in accordance with the Declaration of Helsinki, after the nature of the procedures involved had been explained to them. Participants received $40 €$ upon completion of the experiment.

\section{Stimuli and tasks}

Experimental casks were modelled on a letter-location paradigm developed by Prabhakaran et al. (Prabhakaran et al., 2000) and adapted by Elsley and Parmentier (Elsley and Parmentier, 2009) consisting of a visual memory array of four consonants displayed in four locations (Fig. 1). The verbal stimuli comprised a set of eight consonants (Arial font; $\mathbf{4 8} \mathbf{p t}$ ), selected so as to differ in appearance between upper-and lower-case forms (D, F, H, J, N, Q R, T). The spatial stimuli were presented within a set of eight spatial locations, marked by squares, placed equidistantly in a circular manner at an eccentricicy of $3^{\prime} 63^{\circ}$ from the center of the squares to fixation cross. Both tasks used identical stimuli, but differed with respect to the feature to be attended and memorized. In the verbal task, participants were asked to remember the identicy of the consonants, irrespective of their location. In the spatial cask, participants were instructed to remember the location of the consonants while ignoring their identity. Each task began with a self-paced set of instructions, and five practice trials. At the beginning of each trial, participants first saw a $500-\mathrm{ms}$ central fixation cross, followed by the sample memory array consisting of four consonants, displayed in white (against a black background) and in upper-case, selected at random (without replacement) from the above set of eight. Each consonant appeared in a distinct location randomly selected (without replacement) from the possible set of eight. The letters were presented within a $1^{\prime} 87^{\circ} \times 1^{\prime} 87^{\circ}$ white frame to reduce variations in spatial configuration caused by the consonants per se (Delvenne et al., 2002). The to-be-remembered array remained on the screen for $2000 \mathrm{~ms}$. After a $1200 \mathrm{~ms}$ delay interval, for both conditions, participants were presented with a single lower-case letter in a location for $1000 \mathrm{~ms}$, during which they were required to respond (a $1000 \mathrm{~ms}$ blank screen followed this response period before the onset of the next trial). The task in the verbal condition was to indicate, by button press, whether the consonant had been presented in the to-be-remembered array, regardless of its location. In the spatial task, participants had to decide whether the location had been occupied in the to-be-remembered array, regardless of the identity of the consonant inside. Two types of positive-recognition probes (requiring a "yes" response) were presented, a consonant in its original location (incact probe), and a recombination of a consonant and a location presented in that trial but not together (recombined probe). The negative-recognition probes (requiring a "no" response) were as follows: a new consonant in a new location (in both the verbal and the spatial casks); an old consonant in a new location (in the spatial cask only); and an old location occupied by a new consonant (in the verbal cask only). In both tasks, instructions emphasized the importance of both accuracy and speed.

\section{Procedures}

MEG scans were obtained during the verbal and the spatial tasks. A total of 480 trials were presented in each task. For counterbalancing purposes the order of presentation of the tasks was controlled. Experimencal conditions were completed in different sessions lasting 45 min approximately, separated by a 10 - to 15 -min resting interval. Tasks were purpose-written for the experiment using E-prime (Schneider et al., 2002).

The stimuli were projected through a LCD video-projector (SONY VPL-X600E), situated outside the shielded room on-to a series of inroom mirrors, the last of which was suspended approximately $60 \mathrm{~cm}$ above the subject's face $\left(19^{\prime} 8^{\circ} \times 26^{\prime} 1^{\circ}\right)$.

\section{Data collection and analysis}

All MEG recordings were carried out using a whole-head neuromagnetometer containing an array of 148 magnetometers (4D WHS 2500®, San Diego) and situated in a magnetically shielded room. The data were collected using a sample rate of $254 \mathrm{~Hz}$ and band pass filtered between 0.1 and $50 \mathrm{~Hz}$. MEG daca were submitted to an interactive noise reduction procedure that aided in reducing environmental noise part of the signal analysis package. Vertical and 
horizontal bipolar electro-oculograms (EOG) were also recorded by bipolar montages using a Synamps amplifier (NeuroScan, El Paso. Texas) with $\mathrm{Ag} / \mathrm{AgCl}$ electrodes (same sample rate and online filters as mentioned previously). Trials containing eye movement or blinks (as indicated by peak-to-peak amplitudes in the EOG channels in excess of $50 \mu \mathrm{V}$.) or other myogenic or mechanical artifacts were removed using the automated artifact rejection algorithm implemented in the Brain Electrical Source Analysis software suite (BESA 5.1; Megis Sofware). Only the retention period of trials with correct responses (hits and correct rejections) were included for subsequent analyses.

Digitized MEG daca were imported into MATLAB Version 7.4 (Mathworks, Natick, MA) for analysis using the Fieldtrip software package (http://www.ru.nl/fcdonders/fieldtrip/) and custom-written scripts. Time-frequency (TF) represencation of MEG data was calculated on a single trial basis for a $1700 \mathrm{~ms}$ time window starting from $500 \mathrm{~ms}$ before the onset of the stimulus presentation, and $1200 \mathrm{~ms}$ starting from the beginning of maintenance period using a multi-taper Fourier transform approach applied to short sliding time windows ( $50 \mathrm{~ms}$ ). We used adaptive Hanning tapers of four cycles, with frequencies ranging from 4 to $50 \mathrm{~Hz}$, in $1-\mathrm{Hz}$ steps (de Lange et al., 2008).

As dipolar magnetometer topographies are difficult to interpret with respect of the underlying generator sources, we decided to model frequency changes in the source space (Fan et al., 2007). As stated by Liljestrom et al., (2005) "Localization of ongoing oscillatory activity is important for establishing the normal spatial and spectral variation of cortical rhythmicity in the healthy human brain." In order to construct the source image of the TF data a minimum-norm estimation procedure (MNE) was applied to estimate the cortical origin of the retention period neuronal response. A tessellated cortical mesh template surface derived from the Montreal Neurological Institute (MNI) phantom brain and implemented in SPM5 (http://www.fil.ion.ucl.ac.uk/spm/software/spm5/) served as a brain model to estimate the current source distribution. This MNI dipole mesh ( 3004 nodes) was used to calculate the forward solution using a head model based on overlapping local spheres (Huang et al., 1999). The inverse solution was calculated by applying I2 MNE, with standard Tikhonov regularization, implemented in BrainStorm (http://neuroimage.usc.edu/brainstorm/). Tikhonov regularization is needed to control the effect of the noise on the solution (Bouhamidi and Jbilou, 2007). Jensen and Vanni (2002) have demonstrated that transforming the real and imaginary parts of the Fourier components in the source domain by means of MNE and combining them it is possible to identify source areas of rhythmic activicy in the frequency domain. Accordingly, in our study the underlying current source density (the source strength at each node of the MNI phantom brain) of four frequency bands (theta, $4-8 \mathrm{~Hz}$; alpha, 8-12 Hz; beca, $13-30 \mathrm{~Hz}$; gamma, $30-50 \mathrm{~Hz}$ ) was estimated by calculating the MNE in the frequency domain (Jensen and Vanni, 2002; see also Moratti et al., 2008). Here, the real and imaginary parts of each Fourier component averaged within each of the four frequency bands was submitted to the MNE analysis. Thereafter, the MNEs of the real and imaginary parts were combined by using the root square of the sum of squares of the two Fourier parts as an estimate of absolute amplitude. The change in amplitude was calculated with respect to a baseline period before the beginning of each trial. For each frequency band, the mean time-frequency amplitude of the prestimulus period (between 500 and $0 \mathrm{~ms}$ before stimulus onset) was considered as a baseline and subtracted from the time-frequency representation in order to normalize it. Data were log-transformed in order to reduce the effect of the non-Gaussian distribution (Pivik et al., 1993). The log transformed amplitude values at each dipole location of the brain surface mesh corresponding to the TF-values were scatistically analyzed by using a Student's t-test to compare the verbal and spatial conditions.
Associated $p$-values were thresholded at $p<0.001$ (uncorrected) (see Brookes et al., 2005; Busch et al., 2006; Kilner et al., 2009; Kim and Chung, 2008; Tsuchiya et al., 2008; Van Snellenberg et al., 2007 for a similar statistical approach).

\section{Results}

\section{Behavioral data}

Data from three participants contained MEG signals with noise levels that prevented further analysis. Consequently, these data were excluded from behavioral and T-F analysis. Performance was assessed in terms of accuracy (\% correct) and reaction time (RT) for both positive probe trials (intact and recombined) in each task.

In the verbal cask, participants were more accurate for intact $(M=89.78 \%$ \% $S D=8.61)$ relative to recombined probe trials $(M=83.67 \% ; S D=7.17) \quad\left(t_{10}=3.46, p<0.01\right)$. Additionally, response latencies in the verbal task were also significantly faster on intact probe trials $(M=908.36 \mathrm{~ms} ; S D=163.67)$ relative to recombined probe trials $(M=927.27 \mathrm{~ms} ; S D=158.21)\left(t_{10}=2.60\right.$, $p<0.05)$. In contrast, in the spatial task, accuracy measures for intact $(M=90.59 \% ; S D=8.73)$ and recombined probe trials $(M=89.18 \% ; S D=8.51)$ did not show significant differences $\left(t_{10}=1.32, p>0.20\right)$, and did not differ in terms of RT measures (incact: $M=859.36 \mathrm{~ms} ; S D=224.60$; recombined: $(M=850.54 \mathrm{~ms}$; $S D=224.85)\left(t_{10}=0.92, p>0.30\right)$.

In sum, and in accordance with past research, statistical analyses of our behavioural data indicated verbal-spatial binding effects when consonant identity was task-relevant, but no such effect when spatial location was the task-relevant feature. Based on these observations, we compared the distributed source localization of oscillatory activity between both tasks (Fig. 1).

\section{Distributed source localization of oscillatory activity duting maintenance period}

\section{Theta frequency band $(4-8 \mathrm{~Hz})$}

Higher T-F source amplitudes in the verbal cask as compared to the spatial cask were observed in anterior brain regions, specifically left premotor area between 100 and $150 \mathrm{~ms}\left(t_{10}=4.99, p<0.0005\right.$, all reported t-values correspond to the maximum t-value observed within a significant source cluster) and left dorsolateral prefrontal cortex between 350 and $400 \mathrm{~ms}\left(t_{10}=4.95, p<0.001\right)$. The topographical distribution of the source estimates of rhythmic activity in the theta frequency range revealed that froncal activicy was only greater during the verbal task as compared to the spatial task. Distinct posterior parietal regions also showed greater theta oscillatory activity in the verbal task between $750-800 \mathrm{~ms}\left(t_{10}=5.36\right.$, $p<0.0005), 850-900 \mathrm{~ms}\left(t_{10}=7.67, p<0.0001\right)$ and $1150-1200 \mathrm{~ms}$ $\left(t_{10}=4.93, p<0.001\right)$. Greater activity was also observed over posterior parietal regions in the spatial task as compared to the verbal task between 100 and $150 \mathrm{~ms}\left(t_{10}=6.42, p<0.0001\right), 150$ $200 \mathrm{~ms}\left(t_{10}=7.04, p<0.0001\right), 950-1000 \mathrm{~ms}\left(t_{10}=4.75, p<0.001\right)$ and $1150-1200 \mathrm{~ms}\left(t_{10}=4.73, p<0.001\right)$ (see Figs. 2 and 3 upper panels).

\section{Alpha frequency band (8-12 Hz)}

Condition effects observed in left anterior regions, including premotor areas between 100 and $150 \mathrm{~ms}\left(t_{10}=5.97, p<0.0005\right)$, medial prefrontal area between 200 and $250 \mathrm{~ms}\left(t_{10}=5.93\right.$, $p<0.0005)$ and dorsolateral prefrontal region between 450 and $500 \mathrm{~ms}$ ( $\mathrm{t} 10=4.76, \mathrm{p}<0.001)$, indicated greater oscillatory activity in the alpha frequency range in the verbal task (Fig. 2 mid-upper panel). Distributed source localization of alpha-range activation revealed multiple generators in posterior parietal areas, bilaterally, between 


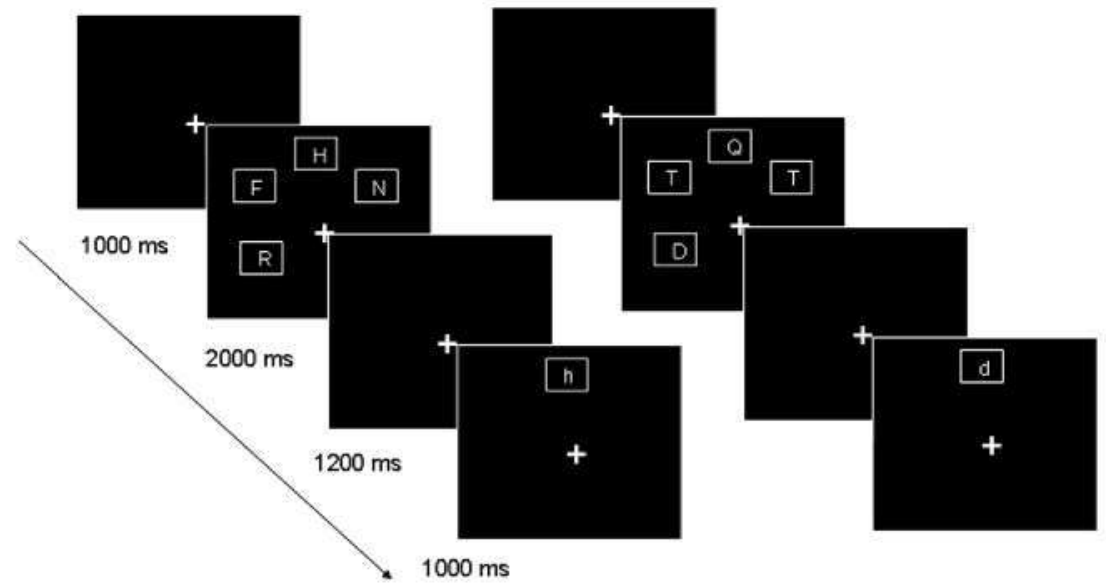

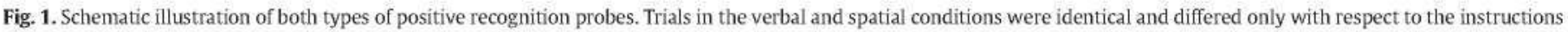

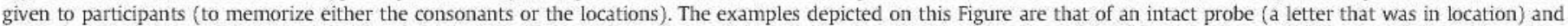
recombined probe (both consonant and location were presented in the to-be-remembered array but not together). Probe consonants were always in lower case form.

250 and $350 \mathrm{~ms}\left(t_{10}=4.65, p<0.001 ; t_{10}=5.61, p<0.0005\right)$ and between 500 and $650 \mathrm{~ms}\left(t_{10}=5.25, p<0.0005 ; t_{10}=4.67, p<0.001\right.$; $\left.t_{10}=5.25, p<0.0005\right)$ during the spatial task as compared to the verbal task. Right premotor area also showed greater oscillatory response during the spatial task between 650 and $700 \mathrm{~ms}\left(t_{10}=5.19\right.$, $p<0.0005$ ) (Fig. 3 mid-upper panel). Again the topographical distribution of the source estimates revealed an effect of greater activity in prefrontal areas during the verbal task. The greater frontal activity during the spatial task was located more posteriorly.

Beta frequency band $(13-30 \mathrm{~Hz})$

Right prefrontal, dorsolateral and ventrolateral regions showed greater beta enhancement during the verbal task between 100 $150 \mathrm{~ms}\left(t_{10}=5.62, p<0.0005\right)$ and $500-550 \mathrm{~ms}\left(t_{10}=6.20\right.$,

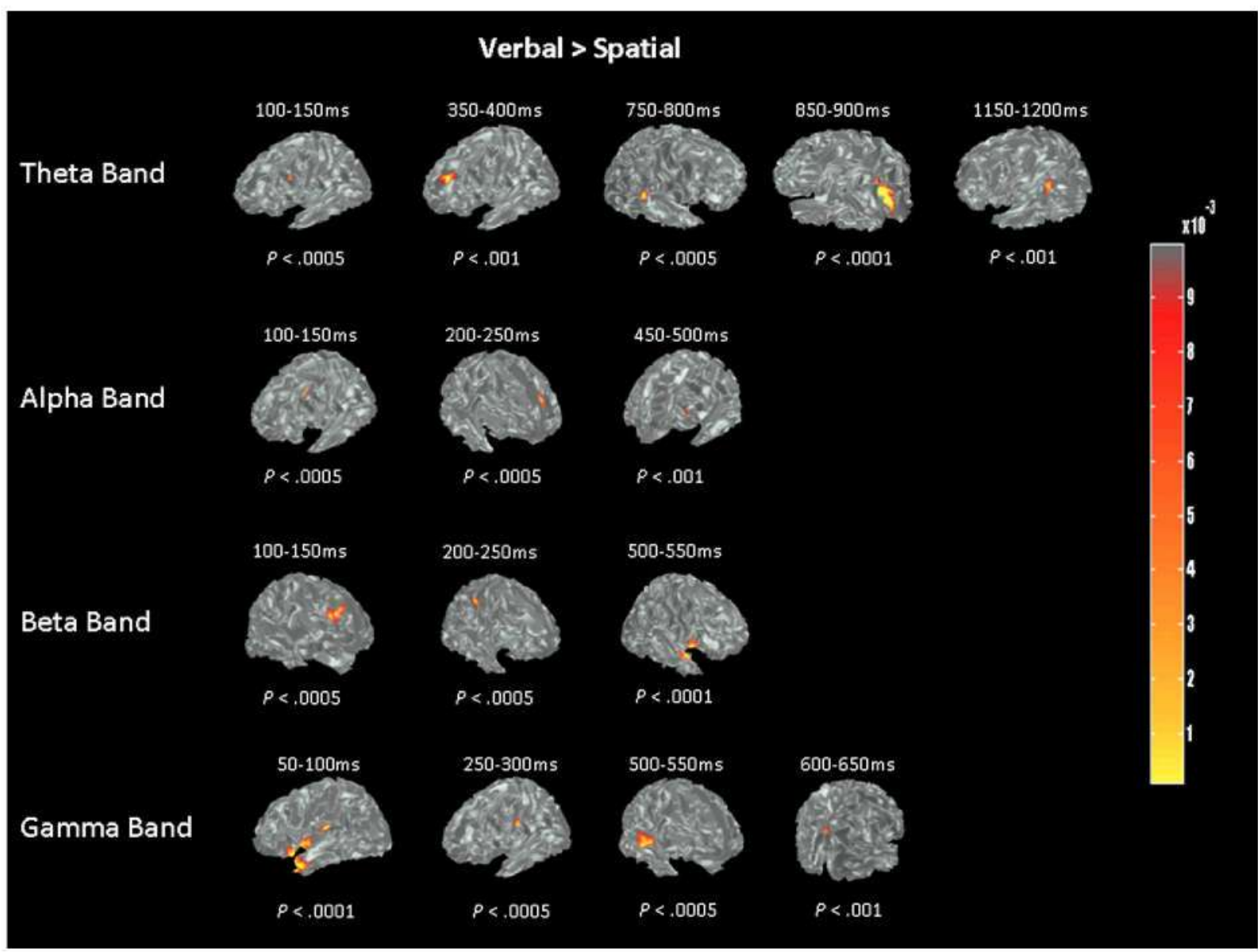

Fig. 2. Shows the results of the group analysis projected onto a tessellated cortical mesh template surface derived from the Montreal Neurological Institute (MNI) phantom brain. The figure depicts cortical source clusters indicating statistically significant increase in amplitude for the verbal task relative to the spatial task in specific time bins for different frequency ranges by means of $p$ values ( $p<0.001$ uncorrected). Only $p$ values exceeding the critical $p$ value of 0.001 are shown. The colorbar indicates the $p$ values. 


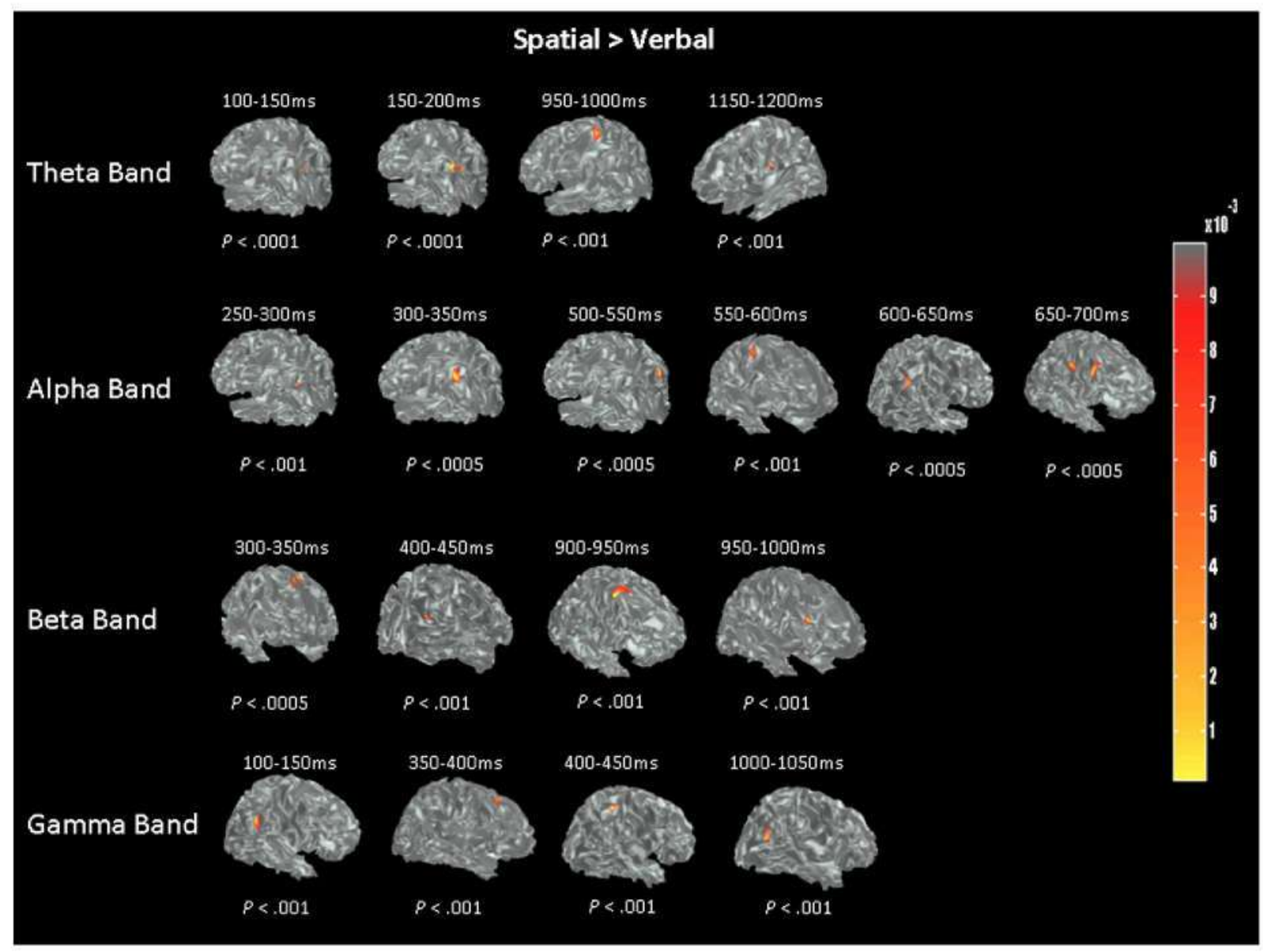

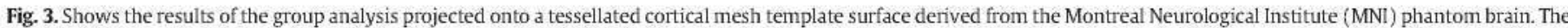

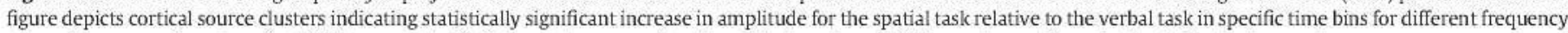
ranges by means of $p$ values ( $p<0.001$ uncorrected). Only $p$ values exceeding the critical $p$ value of 0.001 are shown. The colorbar indicates the $p$ values.

$p<0.0001$ ), respectively (see Fig. 2 mid-lower panel). Additionally, sources located in parietal and temporal areas also exhibited greater oscillatory activity in the beta frequency range in the verbal task as compared to the spatial task between $200-250 \mathrm{~ms}\left(t_{10}=5.33\right.$, $p<0.0005$ ) and between $500-550 \mathrm{~ms}$. Greater activity in this frequency was observed mainly in premotor areas during the spatial task between $300-350 \mathrm{~ms}\left(t_{10}=5.34, p<0.0005\right), 900-950 \mathrm{~ms}$ $\left(t_{10}=4.64, p<0.001\right)$, and $950-100 \mathrm{~ms}\left(t_{10}=4.90, p<0.001\right)$. Additionally, the posterior parietal cortex also showed greater oscillatory activity between 400 and $450 \mathrm{~ms} \quad\left(t_{10}=4.66\right.$, $p<0.001$ ) (see Fig. 3 mid-lower panel). The anterior-prefrontal pattern of activity during the verbal task is also observed in the beta frequency range.

\section{Gamma frequency band $(30-50 \mathrm{~Hz})$}

Topographical distribution of the source estimates for oscillatory activity in the gamma frequency range showed an enhancement over left prefrontal ventrolateral and temporal anterior regions between 50 and $100 \mathrm{~ms}\left(t_{10}=6.62, p<0.0001\right)$, over right posterior temporal region between 500 and $550 \mathrm{~ms}\left(t_{10}=6.08, p<0.0005\right)$, and over posterior parietal cortex between 600 and $650 \mathrm{~ms}$ $\left(t_{10}=4.77, p<0.001\right)$ during the verbal task as compared to the spatial task (Fig. 2 lower panel). Parietal areas showed greater oscillatory activity in the gamma frequency range during the spatial task between $100-150 \mathrm{~ms}\left(t_{10}=4.93, p<0.001\right), 400-450 \mathrm{~ms}$ $\left(t_{10}=4.70, p<0.001\right)$ and $1000-1050 \mathrm{~ms}\left(t_{10}=4.92, p<0.001\right)$, and also premotor areas between 350 and $400 \mathrm{~ms}\left(t_{10}=4.66\right.$, $p<0.001$ ) (Fig. 3 lower panel).

\section{Discussion}

The goal of the current study was to describe the oscillatory activity produced during maintenance of implicit verbal-spatial binding. Our behavioral investigation of spatial (location) versus identity (consonants) maintenance processing, indicated that crosscode binding occurred when spatial information was irrelevant for task completion. In other words, in attending to consonant identity the involuntary binding of spatial location ensued while spatial locations could be attended to in isolation of the consonants occupying them. Consequently, our findings are in agreement with the proposal of an asymmetrical contribution of the spatial dimension previously reported in visual WM studies (Constantinidis and Steinmetz, 2005; Elsley, Parmentier and Maybery, unpublished; Jiang et al., 2000; Mazza et al., 2007; Meegan and Honsberger, 2005). Second, these results indicate that in the absence of explicit processing, cross-code features might be bound and represented in an integrated format (Melcher et al., 2005; O'Craven et al., 1999; Sohn et al., 2004).

Concerning time-frequency analyses of source data, a significant enhancement of neural oscillatory activity was present during the retention period of each task and in all frequency ranges (theta, alpha, beta, and gamma). With a distributed source model we observed activations common to both verbal and spatial tasks engaging distinct parts of the posterior parietal cortex (PPC), mainly in theta and gamma bands. Additional greater activity in the alpha band was observed in PPC during the retention period in the spatial task between 250 and $350 \mathrm{~ms}$ and between 500 and $650 \mathrm{~ms}$. The posterior 
pariecal activity during the retention period of the spatial task is compatible with the proposed role of this area in retaining spatial locations of stimuli (Corbetta et al., 2002; Chafee and Goldman-Rakic, 1998). Along with its involvement in spatial WM, there is a large body of evidence showing that PPC is also engaged in different forms of visual feature integration and cross-modal binding (Martinez et al., 2007; Nobre et al., 2000; Saito et al., 2005; Shafritz et al., 2002; Todd and Marois, 2004; Xu and Chun, 2006; see Macaluso and Driver, 2005 for a review). Our observation of activity over this area in the verbal task is consistent with this view. It has been posited that the parietal lobe cannot be considered as "a unitary entity with a single function" (Jeannerod and Jacob, 2005). Several authors have proposed crossmodal influences of spatial attention on sensory brain regions during multisensory interactions (Macaluso and Driver, 2005; Senkowski et al., 2005). Nonetheless, the critical role of PPC in integrating different features might be independent from spatial processing (Corbetta and Shulman, 2002; Marois and Ivanoff, 2005), and part of its functional role may be to build integrated mental representations of complex stimuli (Marois and lvanoff, 2005). While forming an interesting avenue for future research, the current data do not allow us to elucidate whether the processes supported by the PPC regions activated during the spatial cask are different from those that are engaged during the verbal task.

We also found bilateral effects in theta, beta and gamma band activity over anterior and posterior temporal regions specifically during the verbal task. This pattern of activation could be linked with phonological processing of verbal information (for a review see Gernsbacher and Kaschak, 2003). However, the most interesting finding of the current study is that although differences in activity in all analyzed frequency bands were observed in the verbal and spatial tasks in anterior regions, only activicy in the prefrontal cortex, dorsolateral and ventrolateral, was greater during the verbal task. These differences over prefrontal areas were observed during the first half of the retention period (first $600 \mathrm{~ms}$ ). Anterior sources in the spatial cask were mainly observed in right premotor regions. Indeed, anterior prefrontal areas are considered to be recruited when "selected items become more complex or increase in number" (Corbetta and Shulman, 2002). The precise role of the prefrontal cortex within this task remains open for debate, however. Prefrontal cortex has been proposed as a crucial area in integration processes since it is densely connected with sensory-specific cortices and heteromodal cortical regions (Duncan, 2001; Fuster, 1995). Furthermore, neurons coding different types of information are intermingled in the prefroncal cortex (Petrides and Pandya, 1999; Wilson et al., 1993). Previous work on object-location binding using monkeys concluded that information about object and location converged in the prefrontal cortex, encompassing dorsolateral and ventrolateral surfaces (Rainer et al., 1998a,b; Rao et al., 1997). In humans, greater prefroncal activity during integrated object-location processing has also been shown (Filbey et al., 2005; Mitchell et al., 2000; Simon-Thomas et al., 2003). Moreover, earlier functional neuroimaging (Prabhakaran et al., 2000) and neurophysiological studies (Campo et al., 2005; Wu et al., 2007) in humans have revealed increased activation in prefrontal areas during maintenance of integrated verbal-spatial information. As verbal-spatial binding was observed only in the verbal task it can be inferred that the greater oscillatory activity found in prefrontal areas might be signaling neural processes specifically involved in cross-code binding. However, it is imporcant to highlight that the prefrontal activity in the current study was mainly observed in dorsal and medial regions corresponding to Brodmann's areas (BA) 9 and 46 (see also Campo et al., 2005), while in the study of Prabhakaran et al. this activity was found in BA10. Whether this difference is related to specific mechanisms supporting implicit vs. explicit binding should explored in future studies. Prabhakaran et al (Prabhakaran et al., 2000) suggested that the greater prefrontal activation associated with the integration of verbal and spatial information could be explained by a flexible represencational architecture. However, an alternative interpretation is possible since several authors propose that the prefrontal cortex does not maintain mnemonic representations (Lebedev et al., 2004; Owen et al., 1998; Petrides, 2000; Rissman et al., 2008; Rowe and Passingham, 2001). Instead, prefrontal cortex is considered to participate in controlled processing of the activity of posterior brain regions more directly involved in representing specific features as well as categorical information (Doesburg et al., 2009; Grimault et al., 2009; Kessler and Kiefer, 2005; Mechelli et al., 2004; Rainer and Ranganath, 2002; Serences and Yantis, 2006). This control could be exerted by means of longrange fronto-posterior connections (Grimault et al., 2009; Hasegawa et al., 2000; Kessler and Kiefer, 2005). Models invoking cross-modal influences of spatial attention in multisensory integration include frontal-pariecal interactions as part of the superior attention network (Corbetta and Shulman, 2002; Macaluso and Driver, 2005; Marois and Ivanoff, 2005; Senkowski et al., 2005). Thus, rather than functioning as a storage medium for bound representations, the prefrontal cortex may function as a "pointer" indexing the features that form an object, while the features themselves are recained in more posterior regions. The time window in which we observed greater prefrontal oscillatory activity during the verbal task (0$550 \mathrm{~ms}$ ) is in line with previous results about the maintenance of integrated verbal-spatial represencations (Campo et al., 2005; see also Doesburg et al., 2009). Further, the time range associated with prefrontal oscillatory enhancement is in agreement with a proposal suggesting that processes occurring during this time interval in the information processing pathway could be associated with the escablishment of convergent associations, enabling the integration of different cypes of information into a multimodal representation (Saito et al., 2005; Vogel et al., 2001; Xu and Chun, 2006), which is built and stored in visual WM. Considered together, the current results are consistent with models that suggest a distributed network involving prefrontal and posterior regions, and are in good agreement with the findings of others showing increased fronto-posterior synchronization during verbal-spatial binding (Wu et al., 2007). However, present data do not allow a firm conclusion on whether the activity observed in different frequency ranges bares distinct functional roles in verbal-spatial integration. Oscillatory activicy corresponding to the classical theta, alpha, beta frequency bands, along with gamma band, have been associated with binding processes in previous studies (Bassett et al., 2006; Filbey et al., 2005; Hummel and Gerloff, 2005; Mima et al., 2001; Palva et al., 2005; Wu et al., 2007). Although somewhat speculative, our data might signal of the necessicy of interactions between activities observed across distinct frequencies as a mechanism for the integration of distributed processing (Bassett et al., 2006; Palva et al., 2005).

In conclusion, the present study demonstrated implicit verbalspatial binding effects that were dependent on the cask-relevant feature. Thus, maincaining the identity of verbal information (consonants) arranged in a spatially distributed manner resulted in the concurrent processing of task-irrelevant location information. Critically, the reverse relationship does not hold true supporting the notion of associative asymmetcy. This implicit verbal-spatial binding was linked to a specific effect in oscillatory activity over prefrontal regions in all analyzed frequency ranges during the first half of the retention period and accompanied by greater activity in PPC and temporal regions. We tentatively propose that this network could be the neural basis of the so-called episodic buffer, a component defined as "an interface between a range of systems, each involving a different set of codes" (Baddeley, 2000). However, the episodic buffer would need further elaboration in order to account for the observation of associative asymmetry. Firstly, the buffer in its current form does not allow for implicit binding, since it is under the scrutiny of the central executive and subject to resources available therein. Additionally, it is 
clear that attendance to any object feature is not sufficient to produce the sponcaneous or involuntary binding of all features of that object within the episodic buffer. Which features gain obligatory (or involuntary) access to the episodic buffer and under what circumstances remains to be established.

\section{Acknowledgments}

This work was supported by a research grant from the Spanish Ministry of Education and Science (GrantSEJ2006-14571) to Pablo Campo, by a research grant from the UK's Economic and Social Research Council (GrantRES-062-23-0241) to Fabrice Parmentier and partially supported by a Grant from the Comunidad Autónoma de Madrid (Madr.IB S-SAL-0312-2006). We also acknowledge Angel Nevado for helpful comments on the manuscript.

\section{References}

Avvarez, G.A., Cavanagh, P., 2004. The capacity of visual short-term memory is set both by visual inforination load and by number of objects. Psychol. Sci. 15, 106-111.

Baddeley, A., 1996. The fractionation of working memoly. Proc. Natl. Acad. Sci. U. S. A. 93, 13468-13472.

Baddeley, A, 2000. The episodic butTel; a new component of working meinory? Trends Cogn. Sci. 4, 417-423.

Baddeley, A., Hitcl, G., 1974. Wolking meinory, In: Bower, G.A. (Ed.), The psychology of learning and motivation. Academic Press, New Yolk, Pp. 48-79.

Bao, M., Li, Z.H., Zhang, D.R., 2007. Binding facilitates attention switrhing within working memoly. J. Exp. Psychol. Leann. Mem. Cogn. 33, 959-969.

Bassett, D.S., Meyer-Lindenberg, A., Achard, S., Duke, T., Bullmore, E., 2006. Adaptive reconfiguration of fractal small-world human brain functional networks. Proc. Natl. Acad. Sci. U. S. A. 103, 19518-19523.

Bays, P.M., Husain, M., 2008. Dynamic shifts of limited working memory resources in human vision. Science 321, 851-854

Bodelon, C. Fallah, M., Reynolds, J.H., 2007. Temporal resolution for the perception of features and conjunctions. J. Neurosci. 27, 725-730.

Boulhamidi, A. Jbilou, $K_{n}$ 2007. Sylvester Tikhonow-regularization methods in image restoration. J. Comput. Appl. Math. 206, 86-98.

Brookes, M.J., Gibson, A.M., Hall, S.D., Furlong, P.L, Bames, G.R., Hillebrand, A., Singh, K.D., Holliday, I.E., Francis, S.T., Morris, P.G., 2005. GLM-beamformer method demonstrates stationary field, alpha ERD and gamma ERS co-localisation with IMRI BOLD response in visual cortex. Neuroimage 26, 302-308.

Busch, N.A, Schadow, J., Frund, I., Hermann, C.S., 2006. Time-frequency analysis of target detection reveals an early interface berween bottom-up and top-down processes in the gamma-band. Neuroimage 29, 1106-1116.

Calvert, G.A., Campbell, R., Brammer, M.J., 2000. Evidence from functional magnetic resonance imaging of crossinodal binding in the luminan heteromodal coltex. Curr. Biol. 10, 649-657.

Campo, P., Maestu, F., Ortiz, T., Capilla, A., Santiuste, M., Fernandez, A., Alno, C., 2005. Time modulated prefrontal and parietal activity during the maintenance of integrated information as revealed by magnetoencephalography. Cereb. Cortex 15, 123-130.

Campo, P., Maestu, F., Capilla, A, Morales, M., Femandez, S., del Rio, D., Ortiz, T., 2008. Temporal dynamics of parietal activity during word-location binding. Neuropsychology 22, 85-99.

Constantinidis, C., Steinmetz, M.A., 2005. Posterior parietal cortex automatically encodes the location of salient stimuli. J. Neurosci. 25, 233-238.

Corbetta, M., Shulman, G.L., 2002. Control of goal-directed and stimulus-driven attention in the brain. Nat. Rev. Neurosci. 3, 201-215.

Corbetta, M., Shulınan, G.L., Miezin, F.M., Petersen, S.E., 1995. Superior parietal cortex activation during spatial attention shifts and visual feature conjunction. Science 270. 802-805

Corbetta, M., Kincade, J.M., Shulman, G.L., 2002. Neural systems for visual orienting and their relationships to spatial working meinoly. J. Cogn. Neurosci. 14, 508-523.

Cowan, N., 2001. The indgical number 4 in short-tein memoly: a reconsideration of mental storaze capacity. Behav. Brain Sci. 24, 87-114 discussion 114-185.

Cowan, N., Saults, J.S., Morey. C.C., 2006. Developinent of working memoly for verbalspatial associations. J. Mem. Lang. 55, 274-289.

Chafee, M.V., Goldman-Raleic, P.S., 1998. Matching patterns of activity in primate prefrontal area $8 \mathrm{a}$ and parietal area 7ip neurons during a spatial working memoly task. J. Neurophysiol. 79, 2919-2940.

de Lange, F.P., Jensen, O., Buter, M., Toni, I., 2008. Interactions berween posterior gamma and frontal alpha/beta oscillations during imagined actions. Front Hum. Neurosci. 2, 7 .

Delveme, J.F., Braithwaite, J.J. Humphreys, G.W., 2002. Capaciry limits in visual short term memory for local orientations. Curr. Psychol. Cogn. 21, 681-690.

Doesbuly. S.M., Einberson, LL., Rali, A. Calneron, D., Ward, L.M., 2008a. Asynchrony from synchroly: long-range gamma-band neural synchrouy accolnpanies perception of audiovisual speech asynchrony. Exp. Brain Res. 185, 11-20.

Doesbuly. S.M., Roggeveen, A.B., Kitajo, K., Ward, L.M., 2008b, Large-scale galnina-band phase synchronization and selective attention. Cereb. Cortex 18, 386-396.
Doesburg, S.M., Green, J.J., McDonald, J.J., Ward, L.M., 2009. Rlyythins of consciousiness: binocular rivalry reveals large-scale oscillatory network dynamics mediating visual perception. Plos one 4, e6142.

Domer, T.H., Ketterinam, A., Diesch, E., Ostendorf, F., Villringer, A., Brandt, S.A., 2002 Visual feature and conjunction searches of equal dificulty engage only partially overlapping frontoparietal networks. Neuroimage 15, 16-25.

Duncan, J.r 1984. Selective attention and the organization of visual information. J. Exp Psychol, Gen. 113, 501-517.

Duncan, J., 2001. An adaptive coding model of neural function in prefrontal cortex. Nat. Rev. Neurosci. 2, 820-829.

Elsley, J.V., Parmentier, F.B., 2009. Is verbal-spatial binding in working meinory impaired by a concurrent memory load? Q. J. Exp. Psychol. (Colchester) 1-10.

Eng, H.Y., Chen, D., Jiang. Y., 2005. Visual working memoly for simple and complex visual stimuli. Psychon. Bull. Rev, 12, 1127-1133.

Engel, A.K., Fries, P., Singer, W., 2001. Dynamic predictions: oscillations and synchrony in top-dowl processing. Nat. Rev. Neurosci. 2, 704-716.

Fan, J., Byrne, J., Worden, M.S., Guise, K.G., McCandliss, B.D., Fossella, J., Posner, M.L. 2007. The relation of brain oscillations to attentional nerworks. J. Neurosci. 27. 6197-6206.

Filbey, F.M., Holroyd, T., Carver, F., Sunderland, T., Cohen, R.M., 2005. A magnetoencephalograply spatiotemporal analysis of neural activities during feature binding. Neuroreport 16, 1747-1752.

Friedınan-Hill, S.R., Robertson, L.C., Treisman, A. 1995. Parietal contributions to visual feature binding: evidence from a patient with bilateral lesions. Science 269 , $853-855$.

Fuster, J.M., 1995. Mennory in the cerebral cortex. MIT Press, Cambridge.

Gemsbacher, M.A. Kaschak, M.P., 2003. Neuroimaging studies of language production and comprehension. Annu. Rev. Psychol. 54, 91-114.

Grimault S Robitaille, N Grova, C Lina, JM. Dubarty, A. S Jolicoeur, P, 2009 Oscillatory activity in parietal and dorsolateral prefrontal cortex during retention in visual short-term inemory: additive eflects of spatial attention and memoly load. Hum. Brain Mapp. 30 (10), 3378-3392.

Hasegawa, R.P., Matsumoto, M., Mikami, A., 2000. Search target selection in monkey prefrontal cortex. J. Neurophysiol. 84, 1692-1696.

Hommel, B., Colzato, L, 2004. Visual attention and the temporal dynamics of feature integration. Vis. Cog1. 11, 483-521.

Huang, MX., Masher, J.C., Leahy, RM., 1999. A sensor-weighted overlapping-sphere head model and exhaustive head model comparicon for MEC. Phys. Med. Biol. 44, $423-440$.

Hummel, F., Gerloff, C., 2005. Larger interregional synchrony is associated with greater behavioral success in a colnplex sensoly integration task in humans. Cereb. Cortex $15,670-678$.

Humphrey, G.K., Goodale, M.A, 1998. Probing unconscious visual processing with the Mccollougl effect. Conscious Cogn. 7. 494-519.

Jeannerod, M., Jacob, P., 2005. Visual cognition: a new look at the two-visual system inodel. Neuropsychologia 43, 301-312.

Jensen, $O$. Vanni, S., 2002. A new method to identify multiple sources of ascillatory activity from magnetwencephalographic data. Neuroimage 15, 568-574.

Jiang, Y., Olson, I.R., Chun, M.M., 2000. Organization of visual short-term memoly.J. Exp. Psychol. Leam. Mem. Cogn. 26, 683-702.

Jokisch, D., Jensen, 0., 2007. Modulation of gamma and alpha activicy during a working memory task engaging the dorsal or ventral strealn. J. Neurosci. 27, 3244-3251.

Kahana, M.J., 2006. The cognitive correlates of human brain oscillations. J. Neurosci. 26 1669-1672.

Kahneman, D., Treisman, A., Gibbs, B.j., 1992. The reviewing of object files: objectspecific integration of information. Cogn. Psychol. 24, 175-219.

Kessler, K., Kiefer, M., 2005. Disturbing visual working ineinory: electrophysiological evidence for a role of the prefrontal cortex in recovery from interference. Cereb. Cortex 15, 1075-1087.

Kilner, J.M., Marchant, J.L. Frith, C.D. 2009. Relationship between activity in human primary motor cortex during action obselvation and the inirror neuron system. PLos one 4, e4925.

Kim, JS., Chung, C.K., 2008. Language lateralization using MEG beta frequency desynclironization during auditory oddball stimulation with one-syllable words. Neuroimage 42, 1499-1507.

Kubovy. M., Van Valkenbulg. D., 2001. Auditoly and visual objects. Cognition 80 . 97-126.

Lebedev, M.A, Messinger, A., Kralik, J.D., Wise, S.P., 2004. Representation of attended versus remembered locations in prefiontal cortex. PLoS Biol. 2, e365.

Lee, D., Chun, M.M., 2001. What are the units of visual short-term memory, objects or spatial locations? Percept. Psychophys, 63, 253-257.

Liljestrom, M., Kujala, J., Jensen, 0., Salmelin, R., 2005. Neuromagnetic localization of rhythmic activity in the human brain: a comparison of three methods. Neuroimage $25,734-745$.

Logie, R.H., 1995. Visuo-spatial working memory. Lawrence Enbaum, Hove

Luck, D., Foucher, J.R, OMerlin-Meyer, I., Lepage, M., Danion, J.M., 2008. Assessment of single and bound features in a working memory task in sclizophrenia. Schizophr. Res, 100, 153-160.

Luck, S.J., Vogel, E.K., 1997. The capacity of visual working inemory for features and conjunctions. Nature $390,279-281$.

Macaluso, E., Driver, J., 2005. Multisensory spatial interactions: a window onto functional integration in the human brain. Trends Neurosci. 28, 264-271.

Marois, R, Ivanoח, J., 2005. Capacity limits of information processing in the brain. Trends Cogn. Sci, 9, 296-305.

Martinez-Trujillo, J.C., Treue, S., 2004. Feature-based attention increases the selectivity of population responses in primate visual cortex. Curr. Biol. 14, 744-751. 
Martinez, A., Rainanathan, D.S., Foxe, J.J., Javit, D.C., Hillyard, S.A., 2007. The role of spatial attention in the selection of real and illusory objects. J. Neurosci. 27, $7963-7973$.

Maybely. M.T., Clissa, P.J., Parmentier, F.B., Leung, D., Harsa, G., Fox, A.M., Jones, D.M 2009. Binding of verbal and spatial features in auditory working memory. J. Mem. Lang. 61 (1), 112-133.

Mazza, V., Turatto, M., Umilta, C., Eimer, M., 2007. Attentional selection and identification of visual objects are reflected by distinct electrophysiological responses. Exp. Brain Res. 181, 531-536.

Mechelli, A, Price, C.J., Friston, K.J., Ishai, A., 2004. Where bottom-up meets top-down: neuronal interactions during perception and inagery. Cereb. Cortex 14 , 1256-1265.

Meegan, D.V., Honsberger, M.J., 2005. Spatial information is processed even when it is task-irrelevant: implications for neuroimaging task design. Neuroimage 25. $1043-1055$.

Melcher, D., Papatholnas, T.V., Vidnyanszly, Z, 2005. Implicit attentional selection of bound visual features. Neuron $46,723-729$

Mima, T., Oluwatimilelin, T., Hiraoka, T., Hallett. M., 2001. Transient interhemispheric neuronal synchrony colrelates with object recognition. J. Neurosci. 21. 3942-3948.

Mitchell, K.J., Jolinson, M.K. Raye, C.L., D'Esposito, M., 2000. MMR evidence of agerelated hippocampal dysfunction in feature binding in working memory. Brain Res. Cogn. Brain Res. 10, 197-206.

Mitroff, S.R., Alvarez, G.A., 2007. Space and time, not surface features, guide object persistence. Psychon. Bull. Rev. 14, 1199-1204.

Miyake, A., Shal, P., 1999. Models of Working Melnory. Cambridge University Press, Cambridge.

Moratti, S., Rubio, G., Campo, P., Keil, A., Ortiz, T., 2008. Hypofunction of right temporoparietal cortex during enotional arousal in depression. Arch. Gen. Psychiatry 65, 532-541.

Moley, C.C., 2009. Integrated cross-domain object storage in working inemoly: Evidence from a vertal-spatial memory task Q. J. Exp. Psychol. (Colchester) 1-17

Nobre, A.C., Gitelman, D.R, Dias, E.C., Mesulam, M.M., 2000. Covert visual spatial orienting and saccades: overlapping neural systems. Neuroimage 11, 210-216.

o'Craven, K.M., Downing, P.E., Kanwisher, N., 1999. (MRI evidence for objects as the units of attentional selection. Nature 401, 584-587.

Oberauer, K., Vockenberg, K., 2009. Updating of working memory: lingering bindings. Q.J. Exp. Psychol. (Colchester) 62, 967-987.

Olson, I.R., Marsluuetz, C., 2005. Remembering "what" brings along "where" in visual working memory. Percept. Psychophys. 67, 185-194.

Owen, A.M., Stern, C.E., Look, R.B., Tracey, I., Rosen, B.R., Petrides, M., 1998. Functional organization of spatial and nonspatial working memory processing within the human lateral frontal cortex. Proc. Natl. Acad. Sci. U. S. A. 95, 7721-7726.

Palva, J.M., Palva, S, Kaila, K, 2005. Phase synchrony among neuronal oscillations in the human cortex. J. Neurosci. 25, 3962-3972.

Petrides, M., 2000. Mapping prefrontal cortical systems for the control of cognition. In: Toga, A.W., Mazziota, J.C., Frackowiak, RS. (Eds.), Brain mapping. The systrms. Academic Press, New York, pp. 159-175.

Petrides, M., Pandya, D.N., 1999. Dorsolateral prefrontal cortex: comparative cytoarchitectonic analysis in the human and the macaque brain and corticocortical connection patterns. Eur. J. Neurosci. 11, 1011-1036.

Pivik, R.T., Brougliton, R.J., Coppold, R. Davidson, R.J., Fox, N., Nuwer, M.R., 1993. Guidelines for the recording and quantitative analysis of electroencephalographic activity in research contexts. Psychophysiology 30, 547-558.

Prabhakaran, V., Narayanan, K., Zhao, Z., Gabrieli, J.D., 2000. Integration of diverse information in working inemory within the frontal lobe. Nat. Neurosci, 3, 85-90.

Rainer, G., Rangandth, $C, 2002$. Coding of objects in the prefrontal cortex in inonkeys and humans. Neuroscientist 8,6-11.

Rainer, G. Asaad, W.F. Miller, E.K. 1998d. Memoly fields of neurons in the primate prefrontal cortex. Proc. Natl. Acad. Sci. U. S. A. 95, 15008-15013.

Rainer, G. Asadd, W.F., Miller, E.K., 1998b. Selective representation of relevaln information by neurons in the primate prefrontal coltex. Narure 393, 577-579.

Rao, S.C., Rainer, G., Miller, E.K., 1997. Integration of what and where in the primatr prefrontal cortex. Science 276, 821-824.

Rissman, J., Gazzaley, A, D'Esposito, M., 2008. Dynamic adjustments in prefrontal, hippocampal, and inferior temporal interactions with increasing visual working meinory load. Cereb. Cortex 18, 1618-1629.

Rowe, J.B., Passingham, RE., 2001. Working memory for location and time: activity in prefrontal area 46 relates to selection rather than maintenance in memory. Neuroimage 14, $77-86$.

Saito, D.N., Yoshimura, K., Kochiyama, T. Okada, T., Honda, M., Sadato, N., 2005. Crossmodal binding and activated attentional networks during audio-visual speech integration: a functional MRI study. Cereb. Cortex 15, 1750-1760.
Sauseng, P. Klimesch, W., Doppelmayr, M., Hansimayr, S. Schabus, M., Gruber, W.R. 2004. Theta coupling in the human electroencephalogram during a working memoly task. Neurosci. Lett. 354, 123-126.

Schneider, W., Eschman, A., Zuccoloto, A., 2002. E-Prime reference guide. Psychology Software Tools Inc, Pittsburgh.

Schoenfeld, M.A., Tempelinam, C., Martinez, A., Hopf, J.M., Sattler, C., Heinze, H.J. Hillyard, S.A., 2003. Dynamics of feature binding during object-selective attention. Ploc. Natl. Acad. Sci, U. S. A. 100, 11806-11811.

Scholl, B.J., 2001. Objects and attention: the state of the art. Cognition 80, 1-46.

Senkowski, D., Talsma, D., Hermann, C.S., WoldorI, M.G., 2005. Multisensory processing and oscillatory gamma responses: effects of spatial selective attention. Exp. Brain Res. 166, 411-426.

Senkowski, D., Molholin, S., Golnez-Ramnirez, M., Foxe, J.J., 2006. Oscillatory beta activicy predicts response speed during a inultisensoly audiovisual reaction time task: a high-density electrical mapping study. Cereb. Cortex 16, 1556-1565.

Serences, J.T., Yantis, S., 2006. Selective visual attention and perceptual coherence. Trends Cogn, 5 ci. 10, 38-45.

Shafritz, K.M., Gole, J.C., Marois, R., 2002. The role of the parietal cortex in visual feature binding. Proc. Natl. Acad. Sci. U. S. A. 99, 10917-10922.

Simon-Thomas, E.R., Brodsky, K., Willing, C., Sinha, R., Knigltt, R.T., 2003. Distributed neural activity during object, spatial and integrated processing in humans. Brain Res. Cogn. Brain Res, 16, 457-467.

Singer, W. 1999. Neuronal synchrony: a versatile code for the definition of relations? Neuron 24, 49-65 111-125.

Singer. W., 2001. Consciousness and the binding problem. Aln. N. Y. Acad. Sci. 929 $123-146$.

Singer, W., Gray, C.M., 1995. Visual feature integration and the temporal correlation hypothesis, Almu. Rev, Neulosci. 18, 555-586.

Smith, E.E., Jonides, J., 1998. Neuroimaging analyses of human working memory. Proc. Natl. Acad. Sci. U. S. A. 95, 12061-12068.

Sohn, W., Papathomas, T.V., Blaser, E., Vidnyanszky, Z,, 2004. Object-based cross-feature attentional modulation from color to motion. Vis. Res. 44, 1437-1443.

Tallon-Baudry, C., Berttand, O., 1999. Oscillatory gamma activity in lumans and its role in object representation. Trends Cogn. Sci. 3, 151-162.

Tesche, C.D., Karluu, J., 2000. Theta oscillations index human hippocampal activation during a working memory task. Proc. Natl. Acad. Sci. U. S. A. 97, 919-924.

Todd, J.J., Marois, R, 2004. Capacity limit of visual short-term memory in human posterior parietal cortex. Nature 428, 751-754.

Todd, J.J. Marois, R, 2005. Posterior parietal cortex activity predicts individual differences in visual short-term ineinory capacity. Cogn. Allect. Behav. Neurosci. 5, 144-155.

Treisman, A, 1998. Feabure binding attention and object perception. Philos. Trans. R. Soc. Lond. B Biol. Sci. 353, 1295-1306.

Tsuchiya, N., Kawasaki, H., Oya, H., Howard III, M.A., Adolphs, R, 2008. Decoding face information in time, frequency and space from direct inttacranial recoldings of the huinan brain. PLoS One 3, e3892.

Van Snellenberg, J.X., Whitman, J., McDonald, J.J., Liotti, M., 2007. High temporal resolution imaging of spatial worleing inemoly. Int. Congr. Ser, 1300, 433-436.

Varela, F., Lachaux, J.P. Rodriguez, E., Martinerie, J., 2001. The brainweb: phase synchronization and large-scale integration. Nat. Rev. Neurosci. 2, 229-239.

Vecera, S.P., Faral, M.J., 1994. Does visual attention select objects or locations? J. Exp. Psychol. Gen. 123, 146-160.

Vogel, E.K., Maclizawa, M.G., 2004. Neural activity predicts individual differences in visual working memory capacity. Nature 428, 748-751.

Vogel, E.K., Woodman, G.F., Luck, 5.J., 2001. Storage of features, conjunctions and objects in visual wolking inemoly. J. Exp. Psychol. Huln. Percept. Perform. 27. 92-114

Widinamn, A., Gruber, T., Kujala, T., Tervanieıni, M., Schroger, E., 2007. Binding symbols and sounds: evidence from event-related oscillatory gamma-band activity. Cereb. Cortex. 17, 2696-2702.

Wilson, FA., Scalaidhe, S.P., Goldman-Rakic, P. S., 1993. Dissociation of object and spatial processing domains in primate prefrontal cortex. Science 260, 1955-1958.

Womelsdolf, T., Schoffelen, J.M. Oostenveld, R., Singer, W., Desimone, R., Engel, A.K., Fries, P., 2007. Modulation of neuronal interactions through neuronal synchronization. Science 316, 1609-1612.

Wu, X., Chen, X., Li, Z., Han, S., Zhang, D., 2007. Binding of verbal and spatial information in human working memory involves large-scale neural synchronization at theta frequency. Neuroimage 35, 1654-1662.

Xu, Y., Chun, M.M., 2006. Dissociable neural mechanisms supporting visual short-tem memory for objects. Nature 440, 91-95.

Zhang, W. Luck, 5.J., 2008. Discrete fixed-resolution representations in visual working memory. Nature 453, 233-235. 